\title{
Qualitative Study on Knowledge Sharing by University Hospital Medical Doctors - Focusing on Grounded Theory
}

\author{
Min Sun Kim \\ Assistant Professor, School of Tourism and Distribution Management, College of \\ Business Administration, Hyupsung University, Korea \\ sunnyminkim@hanmail.net
}

\begin{abstract}
This paper utilizes grounded theory in studying the sharing of experiences by a group of university hospital medical doctors. Based on empirical data and the application of knowledge sharing theory, a theoretical framework for a comprehensive approach to knowledge sharing by doctors in the long term is developed.

The 20 participants in this study all have more than 10 years of medical experience and are specialists working in university hospitals in Korea. In addition each participants also hold the position of associate professor or higher at the associated medical school. Four indepth interview and studies were conducted.

For the paradigm model of grounded theory, the concepts were drawn from the causal condition, contextual condition, main context, intervening condition, interaction strategy and result. Low level categories were identified and then high level categories were extracted from them. In this study, 'the process of development through the sharing of experience' is identified as being the key category.

This study is meaningful in that it, unlike existing studies of knowledge sharing, tries to understand and explain the significance and process of knowledge sharing as experienced by medical doctors. While most of the literature about knowledge sharing focuses on a quantitative analysis, this study utilizes qualitative methodology, particularly grounded theory, to understand the substance or meaning the doctors experienced whilst sharing their knowledge. The study suggests how to improve the quality of the knowledge concerned, organize such knowledge and/or advance its utilization and sharing.
\end{abstract}

Keywords: grounded theory, knowledge sharing, professionals, qualitative study, university hospital doctors

\section{Introduction}

Recently, much scholarly work has been done on the sharing of knowledge (Husted \& Michailova, 2002; Liebowitz, 2002). However, these studies were mostly targeted at the general members of enterprises to study the problems of knowledge sharing among individuals or organizations (Bock \& Kim, 2002; Breu \& Hemingway, 2002). Most of these studies focused on the relations or communication among the organization members to understand the factors affecting knowledge interchange or learning (Cook \& Brown, 1999; Pardo et al., 2001). Despite the fact that the sharing of knowledge among special groups of professionals is equally important, relatively few studies have been devoted to this group in particular.

Professionals are defined as those with a specialized or technical education who utilize their knowledge to perform their jobs (Martin \& Shell, 1988). Unlike general organization 
members who work in the type of occupation that requires less specialization, professionals are known to have their own unique behavior (Wallace, 1995). Cho (2001), who diagnosed the professional group pathophysiologically, defined a professional group as a, "group of people with professional spirit based on the professional community". Cho's studies particularly focused on doctors. The community of doctors united in such sprit has the controlling power of the health organization through the ties among the members (Cho, 2001). However, in actual fact the medical professionals working in a specialized treatment domain concentrate only on their specialized area of their duties and tend to be indifferent or uncooperative in duties other than their own treatment field and activities (Seo et al., 2001).In light of this, despite the fact that medical doctors are deluged with a flood of extremely specialized knowledge and information which is in turn becoming ever more fragmented, doctors are still considered a group who mostly utilizes their knowledge for themselves although they do all create and store their knowledge and do not much care about sharing the knowledge and creating larger value. Based on such a background, this study will focus on knowledge sharing in the professional group of medical doctors.

Methodologies of studies on knowledge sharing among professional groups by Fraser et al. (2000) and Jones (2002) mostly emphasized the quantitative correlation. Even when they studied from a qualitative aspect, they were somewhat inadequate in revealing the fundamental understanding of knowledge sharing inherent in the professional group.

This study intends to utilize the grounded theory methodology of Strauss \& Corbin (1998), which is one of the qualitative research methods, in order to understand the meaning and substance of the knowledge sharing experience by observing the knowledge sharing context of medical doctors, who can be considered as a leading profession group. The purpose of this study is advance a theoretical framework for the comprehensive approach to knowledge sharing in the long term by observing and understanding the knowledge sharing experience of the university hospital doctors based on empirical data and by developing a substantive theory of knowledge sharing.

\section{Theoretical Background}

\subsection{Previous Studies of Knowledge Sharing}

Knowledge sharing is an important part of the knowledge management process of combining documented formal data with the implicit knowledge owned by individuals (Liebowitz, 2002). Until recently, studies of knowledge sharing included the study on the interactions between knowledge sharing and the organizational context; study on knowledge sharing, reliability. and compensation system; study on the relations between knowledge sharing and organizational structure; study on information technology and Community of Practice (CoP); study on knowledge sharing focused on general organization members; study on the relationship between the CEO and knowledge sharing; study on organizational cultural factors; study on intention and motivation for knowledge sharing; study on attitude of knowledge initiator and receiver; and study on success and impediment factors.

Most of the previous studies on knowledge sharing attempted to establish the influence of success factors and the impediment or failure factors upon knowledge sharing, and these studies used the quantitative methods to determine and evaluate the static characteristics instead of the knowledge sharing process. As these studies have mostly adopted quantitative approaches, such as studies verifying hypothesis deduced from theories or exploratory studies like case studies or experimental studies, they do not sufficiently explain the experience when the organization members share needed knowledge with colleagues and internal thoughts or 
meaning to individuals. Furthermore, most of the studies on knowledge sharing until now were targeted at general enterprises.

Thus, this study adopted qualitative research methods as it enables the answering of such knowledge sharing process centered questions. Of the many qualitative research methods, the researcher particularly thought that the grounded theory, which studies the multi-dimensional aspects of interactions of human behavior and reveals the interaction process, was appropriate to study the various aspects established during the process of the sharing of medical knowledge by medical doctors, who have been rarely studied until now as either individuals or as members of a group. As such, the purpose of this study is to analyze the relationship between the concepts extracted during the process of knowledge sharing with a focus on the experience of the sharing of knowledge in a professional group and to develop a substantive theory to explain the interaction of situations and behaviors of doctors while sharing such knowledge.

\subsection{Knowledge Sharing by Medical Doctors and Professionals}

As a hospital is organized with professional manpower in many different occupations, there are conflicts among different groups, and professional, administrative and nonprofessional groups are all mixed together. As it is operated 24 hours a day, it is generally very difficult to manage the human resources of the organization. Furthermore, values like service, autonomy, sincerity, justice and confidentiality that the medical professionals pursue can also make knowledge sharing difficult. Doctors are in contact with the patients, the customers, for 24 hours a day to provide direct and indirect medical services and obtain the most information concerning their reactions and requirements and act as the first point of contact with the customers. Therefore, it is very important to systemize doctors' clinical knowledge and know-how as well as sharing and utilizing this.

\section{Research Method}

\subsection{Grounded Theory}

Grounded theory is a philosophical approach developed by Strauss \& Corbin (1998), of the Chicago School. It is a methodology widely used in sociology and is based on symbolic interactions. A symbolic interaction is a unique approach to study the philosophy of both human life and social experience. It has its roots in 'The Discovery of the Grounded Theory' by Glaser and Strauss (Strauss \& Corbin, 1998).

Grounded theory is based on data being systematically collected and analyzed during the research process. It is a method of systematically developing a theory through theoretical sampling, continuous comparison, memo taking, and coding by the researcher with theoretical sensitivity so that the data collection, analysis and final theory during the research period have close correlation (Strauss \& Corbin, 1998).

Theoretical sampling is the extraction of the samples based on a concept by the researcher to search for the dimension or condition to change the attribute of the concept. Continuous comparison, which is considered as the key characteristic of the grounded theory methodology, helps to promote thinking and extract the theoretical samples through the theoretical comparison using comparison, metaphors, and similes of events to clarify these events. A memo taking is a means of recording the thoughts, interpretations, questions, directions, plans and hypotheses that come to the researcher's mind during the study. Coding is the process of deconstructing and conceptualizing the data and it consists of open coding, axial coding and selective coding. Theoretical sensitivity is the researcher's qualification and 
capability to recognize which data is important and give meaning to the data by continuously comparing the data with a skeptical attitude.

\subsection{Seven Steps of Grounded Theory Development}

Grounded theory was so named because it discovers a theory from the data based on actual basis. It is a theoretical development methodology in which each step of development is closely integrated with social research methods in the most advanced and clearly documented ways to develop a thesis and categories. Seven steps of ground theory development are described as follows. First is the step of discovering the research subject. Unlike most deductive research which aims to verify a theory, grounded theory begins without focusing on the research subject. A researcher spends a considerable amount of time mapping out in detail the areas of research before the researchable subject is finally discovered. During that time, the researcher excludes all preconceptions and performs lively research by participating in the life of the subject or at least closely observing. Second is the literature review step. The grounded theorists emphasize that the data in the subject area must be collected first before the literature review. The reason is to ensure that the drawing of categories will not be contaminated by concepts that might be more appropriate in other areas. Third is the sampling step. Instead of random sampling, commonly used in quantitative studies, grounded theory uses theoretical sampling. Theoretical sampling means there is no more categories found from the collected data, i.e. saturation of categories. The saturation of categories means the patterns or key variables are repeatedly found. Fourth is the coding step. In grounded theory, data collection, coding and analysis are conducted at the same time. Fifth is the memo-taking step. Analytically taking note of the researcher's thoughts of how to integrate the relationships of the data, coding and categories into the eventual key descriptive schema is an important element of the grounded theory method. The goal of the researcher is to develop the theoretical idea underpinning the data as the basis for describing the finally integrated schema in detail. Sixth is the step of establishing the most important analysis item. Establishing the finally integrated scheme means finding a key category through continuous comparison and description. Such an analysis item describes the most common behavior pattern in the specific area and integrates and interacts the highly dense sorting of other lower level categories and theses. Seventh is the step of categorizing the memos to draw an outline. The categorization of memos will create the theoretical outline that will be the basis of the final research report.

\subsection{Theoretical Sampling of Research Subject}

The 20 participants in this study have all had more than 10 years of medical experience and are specialists working in university hospitals in Korea with the position of associate professor or higher at medical schools. Four in-depth studies were performed. The reason participants with 10 years or longer experience were selected is because they have the extensive know-how of treatment, research and education in the medical field.

The data were collected through in-depth interviews and participatory observation. The analysis was performed at the same time as data collection. Open coding was performed according to the qualitative data analysis method developed by Strauss \& Cobin (1998). Axial coding was performed at the same time as coding for the process through the paradigm. In selective coding, the relevance of categories, particularly the key categories, is deduced.

\subsection{Data Analysis}

To describe the circumstances included in the statement, the researcher used the line-byline analysis method which analyzes the interview line by line through the question 'Which abstract concept do I need?' In open coding, the meaningful statements were lined up then the 
concepts were found and named. To understand and analyze the named concepts better, questions were asked and compared.

In axial coding, the attributes and dimensions identified through open coding were described in more detail, and the similarities and differences among the categories were described. Causal conditions, key situations, contextual conditions, conciliatory conditions, interaction strategy and result were integrated using the paradigm, an analytical tool. Coding for the process studied the interactions from the data, tracked the change of situations as time passes, and formulated it into each step.

The first step of selective coding is to determine the key categories. The named key categories were refined to continuously develop the attributes and dimensions. Furthermore, selective differentiated sampling was performed to supplement the weak categories.

Hypothetical correlation describing the context of key categories and how they are connected is established and typological analysis of the repeatedly appearing relationships among the categories is performed.

\section{Result}

\subsection{Open Coding}

For open coding, this study reviewed the interview data in-depth to understand how the doctors shared knowledge and then the concepts were extracted and categorized.

As the paradigm model of the grounded theory, the number of concepts extracted from the causal conditions, key situations, contextual conditions, conciliatory conditions, interaction strategy and result was 252. These concepts were abstracted and deduced into 73 low level categories such as 'quality of the knowledge itself' and 'organization and characteristics of the knowledge'. These low level categories were then integrated into 17 high level categories like 'desire to share the knowledge' and 'sharing of experience'.

\subsection{Axial Coding}

Axial coding is the process of recombining the data deconstructed during open coding. It continues the development of attributes and dimensions, links the categories to the low level categories according to the attribute and dimension, and shows how these categories are crossed and linked using the paradigm model (Strauss \& Corbin, 1998). Figure 1 shows the relation among the causal conditions, situations, contextual conditions, conciliatory conditions, interaction strategy and result based on the paradigm model.

\subsection{Selective Coding}

Selective coding is the last step of the coding process and the process of selecting the key category which is the main context identified from the result. The key category deduced in this study is the 'process of development by sharing the experience'. Based on this, typological deduction is performed through the development of a story outline and statement of hypothetical relations.

\subsection{Evaluation Process in Qualitative Research}

Qualitative research is distinguished from quantitative research in the philosophical background, methodical background, research objective, and evaluation guidelines of the research. This study attempted to increase the reliability and validity from the true value, 
applicability, consistency and neutrality according to the strictness evaluation guidelines by Lincoln \& Cuba (1985).

A true value is equivalent to the internal validity in quantitative research. It is the baseline to evaluate the true value of the perception and experience of the participants. In this study, the participants were shown the interview records and analysis result to check if the statement and analysis result described by the researcher matches the experience of the participant.

Applicability is equivalent to the external validity in quantitative research. It describes how much of the research's result can be applied in the context other than the research situation. In this study, the data were collected and meanings were identified until there were no more new data as the participants' statements were repeated.

Consistency is equivalent to reliability in quantitative research. It is the baseline to evaluate whether the results of data collection and analysis are consistent. For this study, the research method and process of data collection and analysis were described in detail. The experts with extensive experience of qualitative research were asked to evaluate the overall research process and results. The subject categories were corrected to improve the consistency of the study.

Neutrality is equivalent to the objectivity in quantitative research. It means being free from all prejudice in the results of the research. To maintain the neutrality for this study, the researcher continued the recording of preconceptions, assumptions and prejudice in the study from the beginning to completion and took memos of interviews, literature and selfawareness in a conscious attempt to compare and identify these.

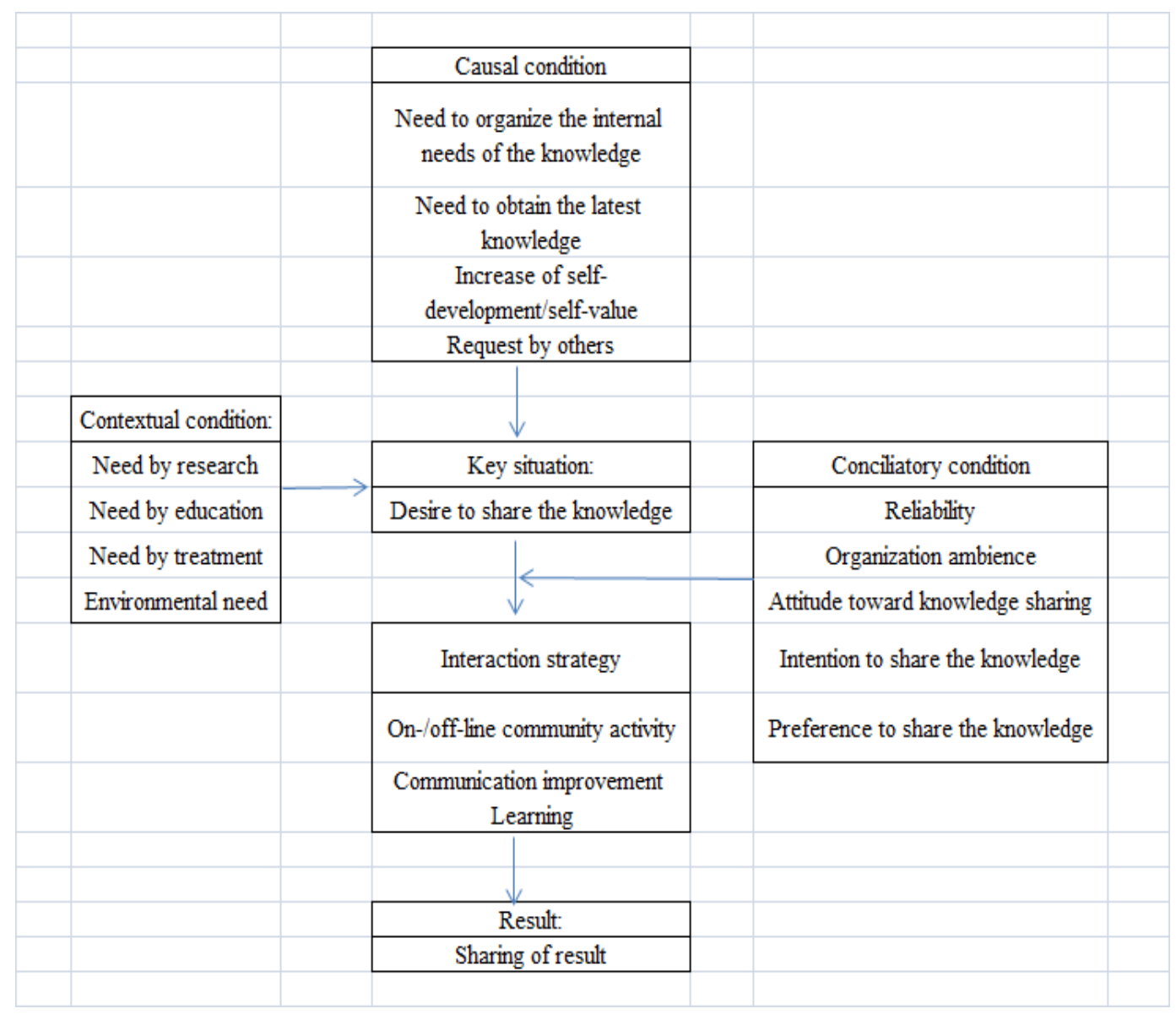




\section{Figure 1. Paradigm Model of Experience of Knowledge Sharing by Doctors}

\section{Conclusion}

This study is meaningful in that it, unlike existing studies of knowledge sharing, tries to understand and explain the significance and process of knowledge sharing experienced by medical doctors. While most of the literature about knowledge sharing focuses on a quantitative analysis, this study utilizes qualitative methodology, particularly grounded theory, to understand in-depth the substance or meaning the doctors experienced whilst sharing their knowledge. The study presents the means to improve the quality of the knowledge concerned, organize such knowledge or advance its utilization and sharing.

Twenty medical specialists at the level of associate professor or higher at domestic university hospitals with 10 years or more experience were invited to participate in four indepth studies on knowledge-sharing among each doctor.

Data were collected through in-depth interviews and participatory observation. The data were analyzed according to open coding, axial coding, and selective coding as suggested by Strauss and Corbin (1998). The open coding process included detailed observations of how doctors share knowledge, after which information was extracted and categorized, and concepts determined. A grounded theory approach was taken through which causal conditions, contexts, central phenomenon, intervening contexts, and interaction strategies were researched, which resulted in the establishment of 252 concepts. The concepts derived were then further abstracted and deduced into 73 low level categories, e.g. quality of knowledge itself, organization and characteristics of knowledge, etc. Some of these low level categories were then integrated into 17 high level categories such as the value of knowledge and the status of the organization.

Analyses of the concepts as well as the low and high level categories of the concepts were undertaken from various perspectives such as causal condition, context, central phenomenon, intervening context, and interaction strategy, with the development process through the sharing of experience emerging as the key central phenomenon.

This study is meaningful as it presents details on how doctors share their knowledge and uses the grounded theory method to discern the meaning of the knowledge-sharing experience, information which can help to improve the quality of knowledge that doctors use and share in their daily lives as medical professionals. This study also offers a theory to explain the central problems and behavioral patterns of knowledge-sharing among doctors. It is hoped that this study will assist hospitals and medical organizations in their planning, development and implementation of knowledge-sharing measures.

\section{References}

[1] G. W. Bock and Y. Kim, "Breaking the Myths of Rewards: An Exploratory Study of Attitude about Knowledge Sharing”, Information Resource Management Journal, http://search.proquest.com.proxy. uhs.ac.kr:8010/docview/215882651 ?accountid=15249, DOI: 10.4018/irmj.2002040102, vol. 15, no. 2, (2002), pp. 14-21.

[2] K. Breu and C. Hemingway, "Collaborative Processes and Knowledge Creation in Communities-ofPractice”, Creativity \& Innovation Management, DOI:10.1111/1467-8691.00247, (2002).

[3] H. G. Cho, "Qualitative Study on Pathological Diagnosis by Professional Group”, Economy and Society, vol. 49, (2001), pp. 341-350.

[4] S. D. N. Cook and J. S. Brown, "Bridging Epistemologies: The Generative Dance between Organizational Knowledge and Organizational Culture”, Organization Science, DOI: 10.1287/orsc.10.4.381, vol. 10, no. 4, (1999). 
[5] V. Fraser, R. Marcella and I. Middleton, "Employee Perceptions of Knowledge Sharing: Employment Threat or Synergy for the Greater Good?”, A Case Study. Competitive Intelligence Review, DOI:10.1002/(SICI)1520-6386(200032)11:2<39::AID-CIR7>3.0.CO;2-J, vol. 11, no. 2, (2000).

[6] K. Husted and S. Michailova, "Diagnosing and Fighting Knowledge Sharing Hostility", Organizational Dynamics, vol. 31, no. 1, (2002).

[7] J. Song, S. Kim and M.-Y. Choi, "Mobile Agent in the U-Medical and Education”, JMUE, ISSN 1975-0080, vol. 1, no. 2, (2006), pp. 1-5.

[8] S. Jones, "Employee Rights, Employee Responsibilities and Knowledge Sharing in Intelligent Organization”, Employee Responsibilities \& Rights Journal, DOI:10.1023/A:1021119503782, vol. 14, no. 2, (2002).

[9] J. Liebowitz, "A Look at NASA Goddard Space Flight Center's Knowledge Management Initiatives", Journal of Computer Information, DOI: 10.1109/MS.2002.1003451, , vol. 19, no. 3, (2002).

[10] Y. S. Lincoln and E. G. Guba, Naturalistic Inquiry, Beverly Hills, CA: Sage Publications, Inc., (1985).

[11] D. D. Martin and R. L. Shell, “Management of Professionals: Insights for Maximizing Cooperation”, Marcel Decker, Inc., PMCID: PMC227318, (1988).

[12] T. A. Pardo, A. M. Cresswell, J. Zhang and F. Thompson, "Interorganizational Knowledge Sharng in Public Sector Innovations”, Academy of Management Proceedings, DOI:10.5465/APBPP.2001.6133798, (2001).

[13] R. S. Tolentino and S. Park, "A Study on U-Healthcare System for Patient Information Management over Ubiquitous Medical Sensor Networks”, IJAST, http://earticle.net/article.aspx?sn=147346, ISSN 20054238, , vol. 18, (2010), pp. 1-10.

[14] Y. J. Seo, D. H. Yang and K. J. Shin, "The Readiness of the University Hospital Employees for the Knowledge-based Management”, Korean Journal of Hospital Management, vol. 6, no. 1, (2001).

[15] S.-S. Kim and Y.-H. Woo, "Ubiquitous Community System for Medical Information", IJMUE, http://www.sersc.org/journals/IJMUE/vol1_no1_2006/5.pdf, ISSN 1975-0080, vol. 1, no. 1, (2006), pp. 1-5.

[16] S. Ullah Khan and A. Ullah Alam, "Knowledge Sharing Management Model (KSMM) for Software Development Outsourcing Vendors”, IJAST, http://www.sersc.org/journals/IJAST/vol45/10.pdf, ISSN 20054238, vol. 45, (2012), pp. 135-144.

[17] A. Strauss and J. Cobin, “Basics of Qualitative Research”, New Deli: Sage Publications, ISBN 5-8360-02991 , (1998).

[18] J. E. Wallace, "Organizations and Professional Commitment in Professional and Nonprofessional Organizations”, Administrative Science Quarterly, vol. 40, (1995).

[19] W. K. Seng, M. H. Kim, R. Besar and F. Salleh, "A Secure Model for Medical Data Sharing”, IJDTA, http://www.sersc.org/journals/IJDTA/vol1_no1/papers/06.pdf, ISSN 2005-4270, vol. 1, no. 1, (2008), pp. 4552.

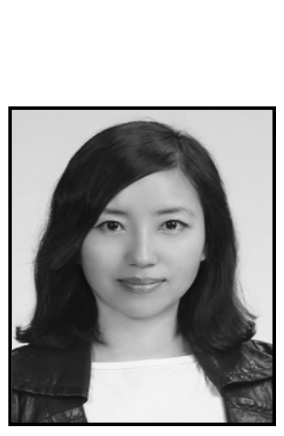

\begin{abstract}
Author
Min Sun Kim, she got her Ph.D. in Management Information System from Ewha Womens University, Korea. She is currently a professor of Department of Tourism and Distribution Management, College of Business Administration, Hyupsung University, Korea. Her current research interests include Smart work, ubiquitous, ebusiness, distribution management including CRM, and Hotel and Tourism management. She can be reached at sunnyminkim@hanmail.net.
\end{abstract}

